\title{
Between heaven and Earth: the exploration of Titan
}

\author{
Tobias C. Owen, ${ }^{a}$ Hasso Niemann, ${ }^{b}$ Sushil Atreya ${ }^{c}$ and Mikhail Y. Zolotov ${ }^{d}$
}

\author{
Received 5th December 2005, Accepted 13th February 2006 \\ First published as an Advance Article on the web 13th July 2006 \\ DOI: $10.1039 / b 517174 a$
}

\begin{abstract}
The atmosphere of Titan represents a bridge between the early solar nebula and atmospheres like ours. The low abundances of primordial noble gases in Titan's atmosphere relative to $\mathrm{N}_{2}$ suggest that the icy planetesimals that formed the satellite must have originated at temperatures higher than $75-100 \mathrm{~K}$. Under these conditions, $\mathrm{N}_{2}$ would also be very poorly trapped and thus Titan's nitrogen, like ours, must have arrived as nitrogen compounds, of which ammonia was likely the major component. This temperature constraint also argues against the trapping of methane. Production of this gas on the satellite after formation appears reasonable based on terrestrial examples of serpentinization, disproportionation and reduction of carbon. These processes require rocks, water, suitable catalysts and the variety of primordial carbon compounds that were plausibly trapped in Titan's ices. Application of this same general scenario to Ganymede, Callisto, KBOs and conditions on the very early Earth seems promising.
\end{abstract}

\section{Introduction}

Eight years ago, at Faraday 109, Owen and Bar-Nun ${ }^{1}$ (hereafter OBN) described a model for delivering volatiles to the inner planets through cometary impacts. The larger context of this model was an investigation of the way in which the major elements reach a planet and what happens to them when they do. Thanks to the Cassini-Huygens mission, we can now test some of the ideas embodied in that model by examining the atmosphere of Titan.

The basic hypothesis of OBN was that icy planetesimals served as a bridge between the interstellar medium and the volatiles we observe today in the inner planet atmospheres. Subsequent study of Jupiter's atmosphere by the Galileo Probe revealed that low temperature icy planetesimals played a key role in bringing heavy elements to Jupiter. ${ }^{2-4}$ As Titan consists of the solar proportion of approximately $50 \%$ ice by mass and thus presumably was built from accreted icy planetesimals, one might expect to find evidence of that icy delivery system on this satellite. The major difference is that Titan's ices probably formed in the sub-nebula surrounding Saturn, whereas the variety of icy planetesimals that putatively brought volatiles to the inner planets and Jupiter should have formed in the solar nebula itself. By tracing the effects of that difference on Titan, however it may be possible to illuminate what happened to the planets. In this essay, we consider the preliminary results from the Huygens probe into Titan's atmosphere in this context, concentrating on the data from the GCMS. ${ }^{5}$

We conclude with applications to other icy objects and the early Earth.

\section{Titan: special properties}

Saturn's giant satellite Titan offers us an opportunity for a kind of cosmic time travel. Any planet or satellite that is within a few AU of its star and small enough for hydrogen to escape into space will

\footnotetext{
${ }^{a}$ Institute for Astronomy, University of Hawaii, 2680 Woodlawn Drive, Honolulu HI 96822, USA. E-mail: owen@ifa.hawaii.edu

${ }^{b}$ NASA/Goddard Space Flight Center, Greenbelt MD, USA. E-mail: hasso.b.niemann@nasa.gov

${ }^{c}$ Atmos Ocean \& Space Sci., University of Michigan, USA. E-mail: atreya@umich.edu

${ }^{d}$ Dept. of Geological Sciences, Arizona State University,USA. E-mail: zolotov@asu.edu
} 
ineluctably become oxidized over time. $\mathrm{OH}$ and $\mathrm{O}$ produced photochemically from water vapor in the atmosphere will attack $\mathrm{CH}_{4}$ and other reduced forms of carbon. The result is a $\mathrm{CO}_{2}$ atmosphere like those of Mars and Venus. The exception to this rule posed by the Earth results from the presence of a huge surface reservoir of liquid water and the emergence of life on our planet. Both of these factors lead to the deposition of $\mathrm{CO}_{2}$ as carbonate rock, while life has obviously contributed our highly anomalous abundant oxygen. At $10 \mathrm{AU}$, the surface temperature of Titan is $94 \mathrm{~K}$; so cold that water vapor is essentially absent from the atmosphere. With no readily available source of oxygen to attack it, $\mathrm{CH}_{4}$ is the second most abundant gas in Titan's atmosphere, with a mixing ratio of $1.6 \%$ in the predominant $\mathrm{N}_{2}$. Molecular hydrogen is present with a mixing ratio of $200 \mathrm{ppm}, \mathrm{CO}$ at $50 \mathrm{ppm}$ and radiogenic argon $\left({ }^{40} \mathrm{Ar}\right)$ at $2.8 \times 10^{-7}$. Trace amounts of several photochemically produced hydrocarbons and nitriles are present in the upper atmosphere (e.g. Raulin, Wilson and Atreya ${ }^{6}$ and Waite et al. ${ }^{7}$ ), where $50 \mathrm{ppb}$ of $\mathrm{CO}_{2}$ is created by reactions between $\mathrm{CO}$ and infalling $\mathrm{H}_{2} \mathrm{O}$ (Samuelson et al. ${ }^{8}$ ). Thus, we find a world literally frozen in time, where we can study chemical and physical processes that may have been important during our planet's earliest history. It is in this sense that Titan lies somewhere between heaven and Earth in the continuum of celestial objects. We may therefore hope to use the atmosphere of Titan to help us bridge the gulf between our own highly evolved planet and the solar nebula from which we came. We consider here the two major constituents, nitrogen and methane.

\section{Nitrogen}

Titan is the only world in our planetary system besides the Earth with a thick nitrogen atmosphere. In fact, there is 12 times more nitrogen above each square centimetre on Titan's surface than exists in a comparable column on Earth. The corresponding surface pressure is 1.5 bars and this is still only $\sim 1 / 5$ of the nitrogen the satellite has produced over geologic time, judging from the depletion of ${ }^{14} \mathrm{~N}$. On Titan, ${ }^{14} \mathrm{~N} /{ }^{15} \mathrm{~N}=183 \pm 5{ }^{5}$ compared with 272 in our atmospheric nitrogen, which matches the majority of values found in meteorites. ${ }^{9}$ The depletion of the light isotope signals extreme atmospheric escape, as previously observed on Mars. ${ }^{10}$ How did this satellite acquire more atmospheric nitrogen than the much more massive Earth?

There are basically two possibilities for the source of $\mathrm{N}$ : either it arrived as $\mathrm{N}_{2}$ or as nitrogen compounds. In the ISM $\sim 90 \%$ of $\mathrm{N}$ is assumed to be in the form of $\mathrm{N}$ and $\mathrm{N}_{2}$ (e.g. ref. 11) hence, $\mathrm{N}_{2}$ is thought to have been the dominant form of nitrogen in the outer solar nebula. This assumption has been substantiated by the discovery that the nitrogen on Jupiter arrived in the form of $\mathrm{N}_{2}$, since ${ }^{15} \mathrm{~N} /{ }^{14} \mathrm{~N}=2.3 \pm 0.3 \times 10^{-3}$ on this giant planet, compared with $3.7 \times 10^{-3}$ on Earth. The low value indicates the homonuclear molecule as the source of the element (Owen et al., ${ }^{9}$ Abbas et al., 2004, Fouchet et al., 2004). Knowing the isotope ratio for nebular $\mathrm{N}_{2}$, we can see that Earth's nitrogen must have been delivered in the form of nitrogen compounds as the presence of nitrogen with the same isotopic ratio in meteorites indeed implies.

We cannot use the isotope trick to elucidate the origin of Titan's nitrogen, however, because of the fractionation we have already described. Instead we turn to an indirect argument involving the noble gases.

$\mathrm{N}_{2}$ is a highly volatile gas. In order to trap large quantities in a solid matrix, temperatures below $40 \mathrm{~K}$ are required regardless of the trapping mechanism $(\mathrm{OBN})$. At such temperatures, large quantities of primordial argon are also trapped, so we would expect a ratio of ${ }^{14} \mathrm{~N} /{ }^{36} \mathrm{Ar}=\mathrm{a}$ few hundred at most, the exact ratio depending on the temperature at which trapping occurred (OBN) and the exact amount of $\mathrm{N}$ that has escaped from Titan. Instead we find a mole fraction of $2.8 \times$ $10^{-7}$ for ${ }^{36} \mathrm{Ar} .{ }^{5}$ We therefore conclude that despite its radically different surface chemistry, Titan acquired its nitrogen in the form of compounds, as did the Earth. We might then expect that the $\sim 50 \%$ of the mass of Titan that is ice is responsible for the huge nitrogen atmosphere: the ice carried the nitrogen to the satellite in the form of compounds trapped in the icy planetesimals that accreted to produce Titan. In contrast, the predominantly rocky Earth had to rely on the aforementioned icy delivery system plus what little was adsorbed in the rocks to supply its meagre supply of volatiles $(\mathrm{OBN})$.

We can assume that the dominant nitrogen compound originally trapped in Titan's ice was ammonia, as it is in comets (where it is also trapped in ice) and the ISM and that the abundance in the ice was close to the cometary abundance of $\sim 1.5 \%$ relative to $\mathrm{H}_{2} \mathrm{O} .{ }^{12}$ Then we can calculate the 
maximum initial amount of nitrogen in the satellite that could have been available to form the atmosphere: $9 \times 10^{23} \mathrm{~g}$. This may be compared to the pre-escape mass of the atmosphere: $4.5 \times$ $10^{22} \mathrm{~g}$. We see that under these assumptions, the icy planetesimals that formed Titan could have easily supplied the amount of nitrogen we now find in the atmosphere. It was simply necessary to have a period early in the satellite's history that was warm enough to allow the $\mathrm{NH}_{3}$ to be vaporized; photochemistry would then convert the $\mathrm{NH}_{3}$ to $\mathrm{N}_{2}{ }^{13}$

There is another important message here. It is reasonable to assume that almost all of the nitrogen that was originally carried to Titan is now in the atmosphere, as is the case on Earth. This assumption is supported by the high degree of isotope fractionation that we observe, which argues against a steady contribution of nitrogen leaking out to the atmosphere that would maintain isotopic normality. Then the original supply of $\mathrm{NH}_{3}$ in the ice was not $\sim 1.5 \%$ but probably closer to $\sim 0.1 \%$. What could have prevented the ice that formed Titan from carrying the same proportion of $\mathrm{NH}_{3} / \mathrm{H}_{2} \mathrm{O}$ that we detect in the ISM and in cometary ice? The answer is again supplied by the extreme depletion of primordial noble gases that we find in Titan's atmosphere today: it must have been the elevated temperature (compared to comet formation temperatures) at which the icy planetesimals accreted in Saturn's subnebula. Alternatively, some part of the missing $\mathrm{NH}_{3}$ may be dissolved in a subcrustal aqueous ocean. In addition to the ammonia, we can assume that some nitrogen was incorporated in the form of organic compounds, such as those found in chrondites (Cronin et al. ${ }^{14}$ ).

\section{Methane}

The present mixing ratio of methane in the atmosphere is $1.6 \times 10^{-2}$ (Niemann et al., ${ }^{5}$ ). Photochemistry is steadily depleting this methane, converting it to aerosols which are precipitating onto Titan's surface. If the current rate of depletion has been constant over geologic time, a layer of photochemical products over $1 \mathrm{~km}$ thick would have been built up over the lifetime of the solar system; continuing the depletion at this rate will lead to the disappearance of the present atmospheric complement in just 10-20 million years. ${ }^{15,16}$ Presumably much of this deposit has been covered by the same surface activity that has obscured the impact craters that must have covered the young surface. Furthermore, unless we just happen to live at the time when an early reservoir of methane is about to disappear, there must be an internal source of this gas to replenish the atmosphere. That this is indeed the case is indicated by the much smaller depletion of the light isotope of carbon compared to ${ }^{14} \mathrm{~N}$ : ${ }^{12} \mathrm{C} /{ }^{13} \mathrm{C}$ in Titan's methane is 82.3 compared to 89.9 on Earth. ${ }^{5}$ Evidently the methane we see today did not experience the same history of fractionation as the $\mathrm{N}_{2}$.

Carbon has a higher abundance than nitrogen in the Sun by about a factor of $4,{ }^{17}$ by about 15 in comets $^{18}$ and $20 \pm 10$ on Earth. ${ }^{19}$ The increase over the solar value results from the difficulty in trapping $\mathrm{N}_{2}$ in solids that we mentioned above, adjusted for the comparable difficulty in trapping $\mathrm{CO}$ and $\mathrm{CH}_{4}$. Unlike nitrogen, most of the carbon in the ISM is in the form of macromolecular or amorphous carbon in grains that are easily trapped in forming icy planetesimals. ${ }^{11}$ We therefore expect a huge reservoir of carbon on Titan, corresponding to the thick nitrogen atmosphere. The amount of precipitated aerosols would not account for this missing carbon, so it must still be in the interior. Detection of ${ }^{40} \mathrm{Ar}$ in Titan's atmosphere, ${ }^{5,20}$ has demonstrated that a pathway exists to allow gases from the interior of the satellite to reach the atmosphere.

Two scenarios have been suggested. Hersant et al. ${ }^{4}$ propose that methane arrived at Titan in the form of clathrate hydrates - crystalline ice in which guest molecules are trapped in the cages formed by the $\mathrm{H}_{2} \mathrm{O}$ - which are now stored at the top of a plausible sub-surface ocean. Owen et al. ${ }^{21}$ suggest instead that the elevated temperatures that inhibited primordial noble gases from being trapped in Titan's constituent icy planetesimals would also have precluded the formation of methane clathrates. Like the noble gases, methane would not have been trapped in the icy planetesimals that formed the satellite. Owen et al. ${ }^{11}$ suggested instead that methane was made in the interior of Titan by a combination of serpentinization and Fisher-Tropsch-like catalytic reactions acting on the trapped $\mathrm{CO}_{2}$ and any surviving primordial organic compounds that brought carbon to the satellite. In this case, the resulting methane could be stored in clathrates made on Titan, or perhaps these coupled processes are still acting today.

We can see these reactions in progress on Earth. In terrestrial oceans, hydration of minerals such as olivine and pyroxene results in the formation of serpentine: $(\mathrm{Mg}, \mathrm{Fe})_{3} \mathrm{Si}_{2} \mathrm{O}_{5}(\mathrm{OH})_{4}$, and molecular hydrogen $\left(\mathrm{H}_{2}(\mathrm{aq})\right)$. In simplified terms, the reaction is $(\mathrm{Mg}, \mathrm{Fe})_{2} \mathrm{SiO}_{4}+11 \mathrm{H}_{2} \mathrm{O} \rightarrow \mathrm{Mg}_{3} \mathrm{Si}_{2} \mathrm{O}_{5}(\mathrm{OH})_{4}$ 
$+\mathrm{Mg}(\mathrm{OH})_{2}+\mathrm{Fe}_{3} \mathrm{O}_{4}+8 \mathrm{H}_{2}(\mathrm{aq})$, i.e., olivine/pyroxene + water yields serpentine + brucite + magnetite + hydrogen. The reaction is exothermic, so once it starts, it can continue as long as fresh supplies of reactants are available. The $\mathrm{H}_{2}(\mathrm{aq})$ so produced is what interests us as it reacts with carbon grains, organic compounds or trapped $\mathrm{CO}_{2}$ in a modified Fischer-Tropsch reaction to produce methane, e.g.:

$$
\mathrm{CO}_{2}(\mathrm{aq})+4 \mathrm{H}_{2}(\mathrm{aq}) \rightarrow \mathrm{CH}_{4}+2 \mathrm{H}_{2} \mathrm{O}
$$

However, the two keys to the efficacy of these two processes in plausible sub-crustal oceans on Titan are the local temperature and the availability of suitable metal catalysts. Kelley et al. ${ }^{22}$ have found methane production in undersea vents (Lost City) on Earth at temperatures of $40-90{ }^{\circ} \mathrm{C}$ and current models of Titan's interior indicate that such temperatures are presently available near the core (C. Sotin, private communication, 2005) This could provide the necessary environment, especially if the boundary between core and ocean were a slurry in which freshly made catalytic material was available. The ${ }^{40} \mathrm{Ar}$ must be reaching the atmosphere from the same region. During and immediately after accretion, the interior would have been warmer, and one might imagine increased production of methane at that time, with subsequent storage in clathrates.

A variation of this scheme has been proposed by Zolotov et al. ${ }^{23}$ again drawing on terrestrial experience (Price and De witt ${ }^{24}$ ). They suggest that disproportionation of carbon by water inside Titan, in which organic matter $+\mathrm{H}_{2} \mathrm{O} \rightarrow \mathrm{CO}_{2}+\mathrm{CH}_{4}+$ intermediate O-bearing organics. Direct oxidation of $\mathrm{H}_{2} \mathrm{O}$ by iron can produce $\mathrm{H}_{2}$, which again converts the $\mathrm{CO}_{2}$ to $\mathrm{CH}_{4}$.

To proceed, one would first like to know whether that ocean really exists, something the Cassini spacecraft should eventually be able to tell us. A further refinement would be a comparison of $\mathrm{D} / \mathrm{H}$ in methane with the value in $\mathrm{H}_{2} \mathrm{O}$. In principle, the value of $\mathrm{D} / \mathrm{H}$ in Titan's methane should be the same as the value in the huge supply of $\mathrm{H}_{2} \mathrm{O}$ ice, once all fractionation effects have been taken into account. In practice, accounting for those effects may be difficult and identifying $\mathrm{H}_{2}$ that has leaked out from the sub-surface reactions may not be possible.

\section{Conclusions}

The interpretation of the low levels of primordial noble gases in Titan's atmosphere as a manifestation of a warm environment $(T \geq 75 \mathrm{~K}$ ) for the formation of constituent planetesimals leads to a requirement for nitrogen to be delivered as compounds - mainly $\mathrm{NH}_{3}$ - and methane to be formed on the satellite. Even the amount of ammonia originally trapped in Titan's ices seems to have been depleted by the locally clement conditions.

This same scenario may explain the absence of Titan-like atmospheres on Ganymede and Callisto. These giant satellites of Jupiter have approximately the same mass and density as Titan, which would suggest that they began with the same composition. One could then reasonably expect similar thick, $\mathrm{N}_{2}$ dominated atmospheres on each of them. But the Jovian sub-nebula from which they formed would have been even warmer than that of Saturn, corresponding to the greater potential energy of the accreting and collapsing material required to make more massive Jupiter. In this case, the amount of ammonia that could be trapped in the icy planetesimals that formed the satellites would have been even less than the already depleted amount we deduced on Titan, and thus perhaps too small to form significant nitrogen atmospheres. However, some methane might have been produced internally from the fraction of carbon compounds that could still have been trapped in these warmer conditions. An early methane atmosphere on Callisto could have produced the dark dust we now find on the surface of the satellite, as irradiation by solar UV and sub-atomic particles in Jupiter's magnetosphere converted the methane to a carbon-rich residue. A. Bazilevsky (private communication 2006) has suggested that this early atmosphere might also have caused the otherwise mysterious degradation of ancient crater rims. Both the dust and the degradation are missing from the more recent, reworked surface of Ganymede, indicating that this transient atmosphere, if indeed it existed, happened very early ( $>$ 4BY ago) in Callisto's history. The absence of detectable (by radio occultation) ${ }^{40} \mathrm{Ar}$ on either satellite implicates particle bombardment as the ultimate remover of tenuous atmospheres.

Similarly, the existence of seemingly fresh methane ice on the surfaces of some of the larger Kuiper Belt Objects appears to require a contemporary source of methane, which otherwise would darken and no longer exhibit the characteristic overtone and combination bands at visible wavelengths that have been observed. 
Finally, we note that this scenario appears to re-open the door to the formation of methane on the earliest Earth by abiogenic processes, such as the serpentinization and Fisher-Tropsch reactions we have described. The icy planetesimals (comets) that impacted the early Earth could have been at least an order of magnitude richer in ammonia than Titan's warmer planetesimals and would also have preserved interstellar organic compounds, as indicated by the isotope anomalies found in IDPs thought to come from comets (e.g., Messenger ${ }^{25}$ ). Trapped $\mathrm{CO}_{2}$ would also have been present. ${ }^{12}$ It remains to be seen what atmospheric lifetime for methane would be possible given the rate of methane production and the UV shielding provided by an early smog layer (Sagan and Chyba $2001^{26}$ ). The recent determination by Tian et $a l .{ }^{27}$ that the rate of hydrogen escape from the early Earth was two orders of magnitude slower than previously thought adds to the likelihood that the methane-ammonia atmosphere originally invoked by Miller $(1953)^{28}$ in his classic experiment demonstrating the first steps of biogenesis was in fact an appropriate model for the early Earth. This unexpected application of our new understanding of Titan merits further examination.

\section{References}

1 T. Owen and A. Bar-Nun, Faraday Discuss., 1998, 109, 453.

2 H. B. Niemann, S. K. Atreya, G. R. Carignan, T. M. Donahue, J. A. Haberman, D. N. Harpold, R. E. Hartle, D. M. Hunten, W. T. Kasprzak, P. R. Mahaffy, T. C. Owen and S. H. Way, J. Geophys. Res., [Atmos.], 1998, 103, 22831.

3 T. Owen, Paul Mahaffy, H. B. Niemann, Sushil Atreya, Thomas Donahue, Akiva Bar-Nun and Imke de Pater, Nature, 1999, 402, 269.

4 F. Hersant, D. Gautier and J. I. Lunine, Planet. Space Sci., 2004, 52, 623.

5 H. B. Niemann, Atreya, S. J. Bauer, G. R. Carignan, J. E. Demick, R. L. Frost, D. Gautier, J. A. Haberman, D. N. Harpold, D. M. Hunten, G. Israel, J. I. Lunine, W. T. Kasprzak, T. C. Owen, M. Paulkovich, F. Raulin, E. Raaen and S. H. Way, Nature, 2005, 438, 779.

6 E. H. Wilson and S. K. Atreya, J. Geophys. Res., [Atmos.], 2004, 1984, 109, DOI: 10.1029/2003JE002181.

7 J. H. Waite, Jr, 2006, in press.

8 R. E. Samuelson, W. C. Maguire, R. A. Hanel, V. G. Kunde, D. E. Jennings, Y. L. Yung and A. C. Aikin, J. Geophys. Res., [Atmos.], 1984, 88, 8709.

9 T. Owen, P. R. Mahaffy, S. Atreya and M. Wong, Astrophys. J., 2001, 553, L77-L79.

10 M. B. McElroy, A. O. C. Nier, A. Wong and Y. Yung, J. Geophys. Res., [Atmos.], 1997, 82, 4341.

11 E. F. van Dishoeck, G. A. Blake, B. T. Draine and J. I. Lunine, in Protostars and Planets III, ed. E. H. Levy and J. I. Lunine, Univ. Arizona Press, Tucson, 1993, 163.

12 D. Bockelee-Morvan, J. Crovisier, M. J. Mumma and H. A. Weaver, in Comets II, ed. M. Festou, H. A. Weaver and U. Keller, Univ. Arizona Press, Tucson, Arizona, 2005, 391.

13 S. K. Atreya, T. M. Donahue and W. R. Kuhn, Science, 1978, 201, 611.

14 J. R. Cronin, S. Pizzarello and D. P. Cruikshank, in Meteorites and the Early Solar System, ed. J. F. Kerridge and M. S. Matthews, Univ. Arizona Press, Tucson, Arizona, 1988, p. 819.

15 D. F. Strobel, Icarus, 1974, 21, 466-470.

16 D. F. Strobel, Planet. Space Sci., 1982, 30, 839-848.

17 N. Grevesse and M. Asplund, EAS Publ. Ser., 2005, 17, 21.

18 J. Geiss, Astronomy, 1980, 1, 1.

19 T. Owen and A. Bar-Nun, Icarus, 1995, 116, 215.

20 J. Hunter Waite, Jr., Hasso Niemann, Roger V. Yelle, Wayne T. Kasprzak, Thomas E. Cravens, Janet G. Luhmann, Ralph L. McNutt, Wing-Huen Ip, David Gell, Virginie De La Haye, Ingo Müller-Wordag, Brian Magee, Nathan Borggren, Steve Ledvina, Greg Fletcher, Erin Walter, Ryan Miller, Stefan Scherer, Rob Thorpe, Jing Xu, Bruce Block and Ken Arnett, Science, 2005, 308, 982.

21 T. Owen, Phys. Usp., 2005, 48, 635-638.

22 Deborah S. Kelley, Jeffrey A. Karson, Gretchen L. Früh-Green, Dana R. Yoerger, Timothy M. Shank, David A. Butterfield, John M. Hayes, Matthew O. Schrenk, Eric J. Olson, Giora Proskurowski, Mike Jakuba, Al Bradley, Ben Larson, Kristin Ludwig, Deborah Glickson, Kate Buckman, Alexander S. Bradley, William J. Brazelton, Kevin Roe, Mitch J. Elend, Adélie Delacour, Stefano M. Bernasconi, Marvin D. Lilley, John A. Baross, Roger E. Summons and Sean P. Sylva, Science, 2005, 307, 1428.

23 M. Y. Zolotov, T. Owen, S. Atreya, H. Niemann and E. L. Shock, EOS Trans, AGU, 2005, 86(52), Fall Mtg. Supp. Abstr. P43B-04.

24 L. C. Price and E. De Witt, Geochim. Cosmochim. Acta, 2001, 65, 3791.

25 S. Messenger, Nature, 2001, 404, 968.

26 C. Sagan and C. Chyba, Science, 1997, 276, 1217-1221.

27 F. Tian, O. B. Toon, A. A. Pavlov and H. De Sterck, Science, 2005, 308, 1014-1017.

28 S. L. Miller, Science, 1953, 117, 528-529. 